BULLETIN OF THE

AMERICAN MATHEMATICAL SOCIETY

Volume 80, Number 4, July 1974

\title{
NONOSCILLATION AND INTEGRAL INEQUALITIES ${ }^{1}$
}

\author{
BY SHMUEL FRIEDLAND
}

Communicated by François Treves, October 22, 1973

1. Introduction. In this note we present a systematical approach to nonoscillation norm conditions for real and complex linear differential systems. We show that the infima of the appropriate integral functionals are constants for nonoscillation criteria. Furthermore in the real case these infima are the best possible nonoscillation constants. Applying an iterative method we prove that the minimal solutions exist and satisfy the Euler-Lagrange equations. This in particular implies that certain first order autonomic systems have periodic solutions. Finally we compute these infima for certain norms. Thus we obtain many known and new results.

2. The variational problems. We consider linear differential systems of the form

$$
x^{\prime}=A(t) x
$$

in some domain $D$. Here $A(t)=\left(a_{j k}(t)\right)_{1}^{n}$ is an $n \times n$ matrix and $x(t)=$ $\left(x_{1}(t), \cdots, x_{n}(t)\right)$ is an $n$ column vector. There are two different cases: (i) $D$ is an interval $[a, b]$. In that case $A(t)$ is real piecewise continuous on $[a, b]$. (ii) $D$ is a simply connected domain on the complex plane with a boundary $\Gamma$. In that case $A(t)$ is a complex valued analytic matrix in $D$. The system (1) is called nonoscillatory [1] (disconjugate [3]) if any nontrivial solution $x(t)=\left(x_{1}(t), \cdots, x_{n}(t)\right)$ of $(1)$ has at least one component $x_{j}(t)$ which does not vanish at any point of $D$. Let $\|x\|_{1}$ and $\|x\|_{2}$ be a pair of norms defined on $R^{n}$. Assume furthermore that each norm is an absolute norm, i.e. $\left\|\left(x_{1}, \cdots, x_{n}\right)\right\|_{j}=\left\|\left(\left|x_{1}\right|, \cdots,\left|x_{n}\right|\right)\right\|_{j}, j=1,2$. Thus these two norms can be naturally extended to $C^{n}$. The matrix norm $\|A\|_{1,2}$ is defined by $\sup \|A x\|_{2} /\|x\|_{1}$. Let $T$ be the collection of all piecewise smooth vectors $x(t)$ on the interval $[a, b]$ which does not vanish at any point of this interval. By $S_{0}$ we denote the set of all $x(t) \in T$ and that

$$
x_{j}(a) x_{j}(b)=0, \quad j=1, \cdots, n .
$$

AMS (MOS) subject classifications (1970). Primary 34C10; Secondary 26A86.

Key words and phrases. Linear systems, nonoscillation, disconjugacy, integral inequalities. 0015 .

${ }^{1}$ Supported in part by the Office of Naval Research Contract N00014-67-A-0112- 
By $S_{a}$ we denote the set of all $x(t) \in T$ such that

$$
x_{j}(b)=-x_{j}(a), \quad j=1, \cdots, n .
$$

Let

$$
\begin{gathered}
c_{1,2}=\inf _{S_{0}} \int_{a}^{b} \frac{\left\|x^{\prime}(t)\right\|_{2}}{\|x(t)\|_{1}} d t, \\
C_{1,2}=\inf _{S_{a}} \int_{a}^{b} \frac{\left\|x^{\prime}(t)\right\|_{2}}{\|x(t)\|_{1}} d t .
\end{gathered}
$$

The constants $c_{1,2}$ and $C_{1,2}$ do not depend on the points $a$ and $b$.

THEOREM 1. Consider the system (1) on a real or complex domain $D$. The following condition implies the nonoscillation of the given system

$$
\int_{a}^{b}\|A(t)\|_{1,2} d t<c_{1,2}
$$

in case that $D=[a, b]$,

$$
\int_{\Gamma}\|A(t)\|_{1,2}|d t|<C_{1,2}
$$

in case that $D$ is a simply connected domain in a complex plane with a boundary $\Gamma$. Moreover in the real case the constant $c_{1,2}$ is best possible.

Assume now that $\|x\|_{i}, j=1,2$, are uniformly Fréchet differentiable norms (UF), i.e. $\|x\|_{j} \in C^{1}\left(S^{n-1}\right)$ for $j=1,2$. By $\|x\|^{*}$ we denote the conjugate norm of $\|x\|$, i.e. $\sup _{y}\left|\sum_{i=1}^{n} x_{i} y_{i}\right| /\|y\|$ and let $\|x\|_{i}$ be the partial derivative $\left(\partial / \partial x_{i}\right)\left\|\left(x_{1}, \cdots, x_{n}\right)\right\|$.

THEOREM 2. Consider the infima (4) and (5). If $\|x\|_{1}$ and $\|x\|_{2}$ are absolute UF norms then both infima are attained. Thus there exist smooth vectors $\xi(t)$ and $\eta(t)$ belonging to the sets $S_{0}$ and $S_{a}$, respectively, such that

$$
c_{1,2}=\int_{0}^{c_{1,2}} \frac{\left\|\xi^{\prime}(t)\right\|_{2}}{\|\xi(t)\|_{1}} d t, \quad C_{1,2}=\int_{0}^{C_{1,2}} \frac{\left\|\eta^{\prime}(t)\right\|_{2}}{\|\eta(t)\|_{1}} d t .
$$

The minimal solutions $\xi(t)$ and $\eta(t)$ satisfy the Euler-Lagrange equations

$$
x_{i}^{\prime}=\|x\|_{1}\|y\|_{2, i}^{*}, \quad y_{i}^{\prime}=-\|y\|_{2}^{*}\|x\|_{1, i}, \quad i=1, \cdots, n,
$$

coupled with the boundary conditions

$$
\xi_{i}(0) \xi_{i}\left(c_{1,2}\right)=0, \quad i=1, \cdots, n, \quad \eta\left(C_{1,2}\right)=-\eta(0) .
$$

Note that $\eta(t)$ is a periodic solution of (9) with the period $2 C_{1,2}$. 
3. Explicit results. In a two dimensional case $x=\left(x_{1}, x_{2}\right)$ the constants $c_{1,2}$ and $C_{1,2}$ are completely determined by the formulas

$$
\begin{aligned}
c_{1,2} & =\int_{0}^{\infty}\left(\|(1, s)\|_{1}\|(s, 1)\|_{2}^{*}\right)^{-1} d s, \\
C_{1.2} & =2 c_{1.2},
\end{aligned}
$$

in the case that $\|x\|_{1}$ and $\|x\|_{2}$ are absolute norms. Let $\|x\|_{p}=\left(\sum_{i=1}^{n}\left|x_{i}\right|^{p}\right)^{1 / p}$ for $1 \leqq p \leqq \infty$. Denote by $c_{p_{1}, p_{2}}(n)$ and $C_{p_{1}, p_{2}}(n)$ the infima (4) and (5) where $\|x\|_{j}=\|x\|_{p_{j}}, j=1,2$, and $n$ is the dimension of the vector $x$. If $1 \leqq p_{2} \leqq p_{1} \leqq \infty$ then

$$
c_{p_{1}, p_{2}}(n)=2 \int_{0}^{1}\left(1+s^{p_{1}}\right)^{-1 / p_{1}}\left(1+s^{\alpha_{2}}\right)^{-1 / q_{2}} d s, \quad p_{2}^{-1}+q_{2}^{-1}=1 .
$$

Furthermore

$$
\begin{aligned}
& c_{p, \infty}(2 m)=2 m^{-1 / p} \int_{0}^{1} {\left[\left(1+s^{p}\right)^{1 / p}(1+s)\right]^{-1} d s } \\
& c_{p, \infty}(2 m+1)=m^{-1 / p} \int_{0}^{1} {\left[\left(s^{p}+\frac{m+1}{m}\right)^{-1 / p}\right.} \\
&\left.+\left(\frac{m+1}{m} s^{p}+1\right)^{-1 / p}\right](1+s)^{-1} d s .
\end{aligned}
$$

The computation of the constants $C_{p_{1}, p_{2}}(n)$ is more difficult. In [5] Schwarz demonstrated that

$$
C_{2,2}(n)=\pi
$$

We show

$$
C_{1,1}(n)=C_{\infty, \infty}(n)=2 \log (n /(n-1))^{n}, \quad n=2,3, \cdots .
$$

This extends some results obtained in [1]-[5].

\section{REFERENCES}

1. Z. Nehari, Oscillation theorems for systems of linear differential equations, Trans. Amer. Math. Soc. 139 (1969), 339-347. MR 39 \#542.

2. - Nonoscillation and disconjugacy of systems of linear differential equations, J. Math. Anal. Appl. 42 (1973), 237-254.

3. B. Schwarz, Norm conditions for disconjugacy of complex differential systems, J. Math. Anal. Appl. 28 (1969), 553-568. MR 40 \#2963.

4. - Mapping of domains by components of solutions of differential systems, J. Differential Equations 10 (1971), 314-323. MR 45 \#621.

5. - Curves on the unit sphere and disconjugacy of differential systems, J. Math. Anal. Appl. 39 (1972), 75-86. MR 46 \#5712.

Department of Mathematics, Stanford University, Stanford, California 94305 